\title{
A Model of Strain Localization and Its Application in Prediction of Shear Bands in Sand
}

\author{
De-Xuan QI ${ }^{1, a *}$, Dong-Li CHEN ${ }^{2, b}$ and Wen-Jie YAN ${ }^{3, c}$ \\ ${ }^{1}$ School of Mechanical Engineering, Hebei University of Technology, Tianjin 300401, China \\ ${ }^{2}$ Tianjin Special Equipment Inspection Institute, Tianjin 300192, China \\ ${ }^{3}$ School of Computer Science and Engineering, Hebei University of Technology, Tianjin 300401, \\ China \\ adxqi@tju.edu.cn, bchendongli860606@163.com, c yanwenjie@scse.hebut.edu.cn \\ ${ }^{*}$ Corresponding author: De-Xuan QI
}

Keywords: Strain localization, Locally-deformed bands, Shear bands, Two-phase equilibrium.

\begin{abstract}
Strain localization in sand is modelled as the event of two-phase coexistence. Shear bands pertain to the high-strain phase of materials. And other regions outside bands pertain to the low-strain phase. Inside each phase the equilibrium equations must be satisfied. Across interfaces between the two phases, discontinuity of displacement gradient and stress is considered. And the continuity of displacement and traction is imposed. The Maxwell relation is also applied. For strain localization under plane strain, four governing equations involving five variables are derived, and the occurrence of locally-deformed bands is attributed to finding a possible minimum of loads. At this load, governing equations have physically acceptable real solutions. Via these solutions, both stresses and strains inside and outside bands and the inclination angle of the band can be all predicted. And the results are agreed well with experiment results.
\end{abstract}

\section{Instruction}

The strain localization phenomenon has been observed at geotechnical structures, and observed in laboratory experiments. Pioneer studies for strain localization in sand have been reported, see the work in the seventies of last century [1-3], later [4-8], and more recently [9-12]. Especially, we refer the reader to the overview article [13].

In the present paper, we would like to provide another mechanism for strain localization. The strain inside the locally-deformed bands is apparently higher than the strain inside other regions outside the bands. That is why the bands are called "Locally-deformed bands", so the particles inside locally-deformed bands pertain to high-strain phase of materials and the ones inside other regions pertains to low-strain phase. That is, two distinct phases of materials can coexist together in some materials under certain conditions. It has been shown that such two-phase equilibrium can occur in the materials with strain-softening behaviour $[14,15]$. In addition, many experiments on sand have revealed that sand with shear bands shows strain-softening behaviour [10].

We focus on finding the two-phase solution for strain localization problems. The interfaces between locally-deformed bands and other regions could be observed clearly in most of specimens with bands, so displacement gradient and stress across the interfaces are generally discontinuous. Under the theory of two-phase equilibrium the possibility of the discontinuity may be considered.

\section{Methods}

\section{Governing Equations for Two-Phase Equilibrium}

A sand specimen under plane strain is shown in Figure 1. The part in shear band is signed by "+" phase. The parts outside the band are signed by "-" phase. In common Cartesian coordinate system, $\left(X_{1}, X_{2}, X_{3}\right)$ is denote the position vector in the reference configuration. And $\left(x_{1}, x_{2}, x_{3}\right)$ is denote the position vector in the deformed configuration. The deformation gradient tensor is defined by 
$\boldsymbol{F}=\partial \boldsymbol{x} / \partial \boldsymbol{X}$. The $\boldsymbol{N}=\left[N_{1}, N_{2}, 0\right]^{\mathrm{T}}$ is the unit vector in the reference configuration as shown in Figure 1 . The inclination angle of the shear band, denoted by $\theta$, is given by

$$
\theta=\tan ^{-1}\left(N_{2} / N_{1}\right) .
$$

The following equilibrium equations must be satisfied

$$
\operatorname{Div} \boldsymbol{\pi}^{\mathrm{T}}=\mathbf{0},
$$

where $\pi$ is the first Piola-Kirchhoff stress tensor. This is two-phase equilibrium required.

For continuum, the continuity of traction across interfaces must be satisfied, namely

$$
[\pi] N=\mathbf{0},
$$

where $[\square]=\square^{\dagger}-\square$ is a jump of a function across an interface.

Thus we apply the continuity of displacements across interfaces. That is, the jump $[\boldsymbol{F}]$ must satisfy Eq. (4) (for the reason see [16]),

$$
[F]=f \otimes N,
$$

where $f$ is a vector and can be written as

$$
f=[F] N .
$$

The Maxwell relation is also imposed as a necessary condition

$$
[W]-\boldsymbol{f} \cdot \pi \boldsymbol{N}=0,
$$

where $W$ is the stress-work function. The Maxwell relation indicated the continuity of traction and displacements across interfaces. It ensures stability with respect to perturbations of interfaces in the reference configuration.

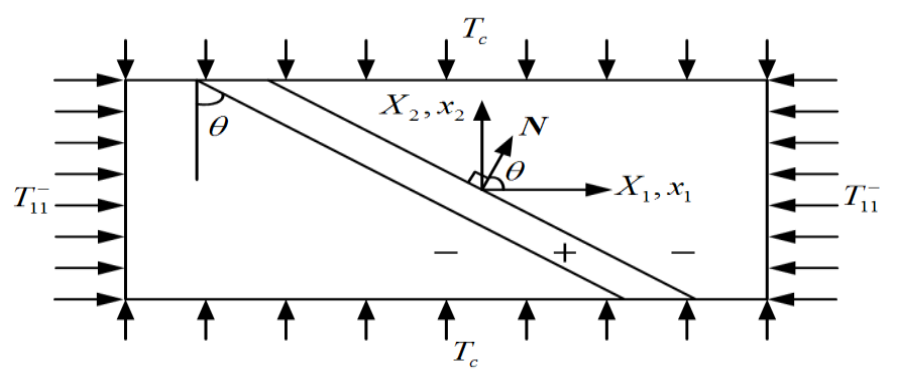

Figure 1. A top view of a specimen under plane strain.

\section{Low-Strain Phase}

The stress field inside the shear band under plane strain, denoted by $\boldsymbol{T}^{-}$, takes the form

$$
\mathbf{T}^{-}=\left[\begin{array}{ccc}
T_{11}^{-} & 0 & 0 \\
0 & T_{c} & 0 \\
0 & 0 & v\left(T_{11}^{-}+T_{c}\right)
\end{array}\right],
$$

where $T_{c}$ is the confining pressure and $v$ is the Poisson ratio.

The deformation gradient for plane strain can be assumed to be

$$
\mathbf{F}^{-}=\left[\begin{array}{ccc}
\lambda_{1} & 0 & 0 \\
0 & \lambda_{2} & 0 \\
0 & 0 & 1
\end{array}\right],
$$

and, from $\boldsymbol{E}=(1 / 2)\left(\boldsymbol{F}^{\mathrm{T}} \boldsymbol{F}-\boldsymbol{I}\right), \lambda_{1}$ and $\lambda_{2}$ can be determined as follows 


$$
\lambda_{1}=\sqrt{1+2 E_{11}^{-}}, \quad \lambda_{2}=\sqrt{1+2 E_{22}^{-}},
$$

The associated strain, denoted by $\boldsymbol{E}^{-}$, is given by

$$
\begin{aligned}
& \mathbf{E}^{-}=\mathbf{M T}^{-} \\
& =\left[\begin{array}{ccc}
-\frac{-T_{11}^{-}+v T_{c}+v^{2} T_{11}^{-}+v^{2} T_{c}}{E} & 0 & 0 \\
0 & -\frac{v T_{11}^{-}-T_{c}+v^{2} T_{11}^{-}+v^{2} T_{c}}{E} & 0 \\
0 & 0 & 0
\end{array}\right],
\end{aligned}
$$

The first Piola-Kirchhoff stress tensor is

$$
\boldsymbol{\pi}^{-}=\mathbf{F}^{-}\left(\mathbf{T}^{-}\right)^{\mathrm{T}}=\left[\begin{array}{ccc}
\lambda_{1} T_{11}^{-} & 0 & 0 \\
0 & \lambda_{2} T_{c} & 0 \\
0 & 0 & v\left(T_{11}^{-}+T_{c}\right)
\end{array}\right],
$$

and the strain energy is given by

$$
\begin{aligned}
& W^{-}=\frac{1}{2} \mathbf{E}^{-} \mathbf{L} \mathbf{E}^{-} \\
& =-\frac{-\left(T_{11}^{-}\right)^{2}+2 v T_{11}^{-} T_{c}+v^{2}\left(T_{11}^{-}\right)^{2}+2 v^{2} T_{11}^{-} T_{c}-\left(T_{c}\right)^{2}+v^{2}\left(T_{c}\right)^{2}}{2 E}
\end{aligned}
$$

\section{Lower Yield Point and Upper Yield Point}

Many experiments have revealed that sand with locally-deformed bands shows strain-softening behavior, see, e.g., the stress-strain curves in the Figure $9(a, b)$ in [10]. For plane strain the stresses at upper yield point and the lower yield point, denoted by $\boldsymbol{T}^{\mathrm{y}}$ and $\boldsymbol{T}^{\mathrm{yL}}$, can be respectively written as

$$
\begin{aligned}
\mathbf{T}^{\mathrm{yU}} & =\left[\begin{array}{ccc}
T_{11}^{\mathrm{yU}} & 0 & 0 \\
0 & T_{c} & 0 \\
0 & 0 & v\left(T_{11}^{\mathrm{yU}}+T_{c}\right)
\end{array}\right], \\
\mathbf{T}^{\mathrm{yL}} & =\left[\begin{array}{ccc}
T_{11}^{\mathrm{yL}} & 0 & 0 \\
0 & T_{c} & 0 \\
0 & 0 & v\left(T_{11}^{\mathrm{yL}}+T_{c}\right)
\end{array}\right],
\end{aligned}
$$

where $T_{11}^{\mathrm{yU}}$ and $T_{11}^{\mathrm{yL}}$ are the stress components in the $X_{1}\left(x_{1}\right)$ - axis direction (compression direction) at upper yield point and the lower yield point, respectively.

The corresponding upper yield strain and lower yield strain, denoted by $\mathbf{E}^{\mathrm{yU}}$ and $\mathbf{E}^{\mathrm{yL}}$, take the following forms, respectively,

$$
\begin{aligned}
\mathbf{E}^{\mathrm{yU}} & =\left[\begin{array}{ccc}
E_{11}^{\mathrm{yU}} & 0 & 0 \\
0 & E_{\mathrm{v}}^{\mathrm{yU}}-E_{11}^{\mathrm{yU}} & 0 \\
0 & 0 & 0
\end{array}\right], \\
\mathbf{E}^{\mathrm{yL}} & =\left[\begin{array}{ccc}
E_{11}^{\mathrm{yL}} & 0 & 0 \\
0 & E_{\mathrm{v}}^{\mathrm{yL}}-E_{11}^{\mathrm{yL}} & 0 \\
0 & 0 & 0
\end{array}\right],
\end{aligned}
$$

where $E_{11}^{\mathrm{yU}}$ and $E_{11}^{\mathrm{yL}}$ are the upper yield strain and the lower yield strain along the $X_{1}\left(x_{1}\right)$-axis direction under plane strain. And $E_{\mathrm{v}}^{\mathrm{yU}}$ and $E_{\mathrm{v}}^{\mathrm{yL}}$ are the volumetric strains at the upper yield point and lower yield point, respectively. 


\section{High-Strain Phase}

For the specimen under plane strain, we can assume that $[\boldsymbol{F}]$ takes the form of

$$
[\mathbf{F}]=\left[\begin{array}{ccc}
f_{1} N_{1} & f_{1} N_{2} & 0 \\
f_{2} N_{1} & f_{2} N_{2} & 0 \\
0 & 0 & 0
\end{array}\right] .
$$

From Eq. (5), we have

$$
\boldsymbol{f}=\left[f_{1}, f_{2}, 0\right]^{\mathrm{T}} .
$$

Then, we have consequently $\boldsymbol{F}^{+}=\boldsymbol{F}^{-}+[\boldsymbol{F}], \boldsymbol{E}^{+}=(1 / 2)\left(\boldsymbol{F}_{+}^{\mathrm{T}} \boldsymbol{F}_{+}-\boldsymbol{I}\right)$, and

$$
\dot{\boldsymbol{E}}=\boldsymbol{E}^{+}-\boldsymbol{E}^{\mathrm{yL}}
$$

In order to obtain $\dot{\boldsymbol{T}}$ in High-strain phase, we use Drucker-Prager constitutive models for finite deformations [17]. The Drucker-Prager constitutive model is suggested initially for infinite deformations. We now broaden it to be available for finite deformations,

$$
\begin{gathered}
\dot{\boldsymbol{T}}=\boldsymbol{L}^{\mathrm{ep}} \dot{\boldsymbol{E}}, \\
L_{i j k l}^{e p}=L_{i j k l}-\frac{G \frac{1}{\sqrt{J_{2}}} s_{i j}+3 \alpha K \delta_{i j}}{9 K \alpha^{2}+G+A}\left(\frac{G}{\sqrt{J_{2}}} s_{k l}+3 K \alpha \delta_{k l}\right),
\end{gathered}
$$

where $L_{i j k l}$ is the fourth-order elastic stiffness tensor, $L_{i j k l}=2 G \bar{I}_{i j k l}+K \delta_{i j} \delta_{k l}, G$ is the elastic shear modulus, $K$ is the bulk modulus, and $\overline{\boldsymbol{I}}$ is the fourth-order special identity tensor $\bar{I}_{i j k l}=(1 / 2)\left(\delta_{i k} \delta_{j l}+\delta_{j k} \delta_{i l}\right)-(1 / 3) \delta_{i j} \delta_{k l} . J_{2}$ is the second stress invariant of the deviatoric stress components. $\delta_{i j}$ is the Kronecker delta, $s_{i j}$ is the components of deviatory stress tensor, $s_{i j}=\mathrm{T}_{i j}-(1 / 3) \mathrm{T}_{k k} \delta_{i j} . A$ is the hardening parameter. $\alpha$ is material constants which are related to

$$
\alpha=\frac{\sin \varphi}{\sqrt{3} \sqrt{3+\sin ^{2} \varphi}},
$$

where $\varphi$ is the friction angle. And then we obtain

$$
\boldsymbol{T}^{+}=\boldsymbol{T}^{\mathrm{Y \perp}}+\dot{\boldsymbol{T}},
$$

and

$$
[\pi]=\boldsymbol{F}^{+} \boldsymbol{T}^{+}-\pi^{-} .
$$

The stress-work function for plastic phase is given by $W^{+}=\int_{0}^{E_{A B}^{+}} T_{A B}^{+}\left(\boldsymbol{E}^{+}\right) d E_{A B}^{+}$. We have

$$
\begin{aligned}
W^{+}= & \frac{1}{2} T_{A B}^{y U} E_{A B}^{y U}+\frac{1}{2}\left(T_{A B}^{y U}+T_{A B}^{y L}\right)\left(E_{A B}^{y L}-E_{A B}^{y U}\right) \\
& +T_{A B}^{y L} \dot{E}_{A B}+\frac{1}{2} \dot{T}_{A B} \dot{E}_{A B}
\end{aligned}
$$

\section{Governing Equations}

Substituting $N=\left[N_{1}, N_{2}, 0\right]^{\mathrm{T}}$ and (24) into the governing Eq. (3). And substituting (11), (12), (19), and (25) into the Maxwell relation (6), we have

$$
q_{1}\left(T_{11}^{-}, f_{1}, f_{2}, N_{1}, N_{2}\right)=0,
$$




$$
\begin{gathered}
q_{2}\left(T_{11}^{-}, f_{1}, f_{2}, N_{1}, N_{2}\right)=0, \\
q_{3}\left(T_{11}^{-}, f_{1}, f_{2}, N_{1}, N_{2}\right)=0 .
\end{gathered}
$$

The above three equations can readily be deduced with the aid of the Mathematica 8.0 software. The two-phase piece-wise homogenous deformations can be in equilibrium only if Eqs. (26)-(28) together with $N_{1}^{2}+N_{2}^{2}=1$ have a unique, real, physically acceptable solution for $\left[N_{1}, N_{2}, f_{1}, f_{2}\right]^{\mathrm{T}}$ when the absolute value of $T_{11}^{-}$attains a minimum. The physically acceptability implies that for the specimen as shown in Figure 1, where $T_{11}^{-}<0, N_{1}>0$ and $N_{2}>0$, the real solution must satisfy $f_{1}<0$ and $f_{2}>0$ in order to ensure that $\left[F_{11}\right]=f_{1} N_{1}<0$ and $\left[F_{22}\right]=f_{2} N_{2}>0$, in other words, the material inside the locally-deformed band should be further compressed.

Eqs. (26)-(28) consist of, or can be transferred to involving only, polynomials for the variables $N_{1}, N_{2}, f_{1}$ and $f_{2}$. They can be solved numerically via the polynomial homotopy continuation (PHC) algorithm.

\section{Results}

As an illustration, the strain localization in sand specimen under plane strain is analyzed. The stressstrain curve of F3-sand in plane strain experiments was given in the Figure 9(b) in [10]. The curve is used to verify our model. By this curve, some values can be easily evaluated as follows: $T_{11}^{\mathrm{yU}}=-$ $112.5 \mathrm{KPa}, E_{11}^{\mathrm{yU}}=-0.02, E_{\mathrm{v}}^{\mathrm{yU}}=0.015, T_{11}^{\mathrm{yL}}=-75 \mathrm{KPa}, E_{11}^{\mathrm{yL}}=-0.04, E_{\mathrm{v}}^{\mathrm{yL}}=0.021$, and $E=5625 \mathrm{KPa}$. And, from the article [10], the friction angle is $\varphi=49.8^{\circ}$ and confining pressure is $T_{c}=-15 \mathrm{KPa}$. The Poisson ratio is 0.27 . With the aid of PHC, numerically solving Eqs. (26)-(28) together with $N_{1}^{2}+N_{2}^{2}=1$ shows that:When $T_{11}^{-}>-92$ there is no real solution. When $T_{11}^{-}<-93$ there are eight real solutions for $\left[N_{1}, N_{2}, f_{1}, f_{2}\right]^{T}$. The number of the real solutions is always multiple of four. The four solutions in each group are in the forms of $\left[N_{1}>0, N_{2}>0, f_{1}<0, f_{2}>0\right]^{\mathrm{T}},\left[N_{1}<0, N_{2}>0, f_{1}>0, f_{2}>0\right]^{\mathrm{T}}$, $\left[N_{1}>0, N_{2}<0, f_{1}<0, f_{2}<0\right]^{\mathrm{T}}$ and $\left[N_{1}<0, N_{2}<0, f_{1}>0, f_{2}<0\right]^{\mathrm{T}}$. All the four solutions satisfy $f_{1} N_{1}<0$ and $f_{2} N_{2}>0$. They are all physically acceptable. Without loss of generality, only the solution $\left[N_{1}>0, N_{2}>0, f_{1}<0, f_{2}>0\right]^{\mathrm{T}}$ is considered in the following analysis.

We choose $-93+\delta_{1}<T_{11}^{-}<-92-\delta_{2}$, where $0<\delta_{1}<1$ and $0<\delta_{2}<1$, and continue to find the solutions. The absolute values of the solutions are closer, while the values of $\delta_{1}$ and $\delta_{2}$ are greater. Finally, we find that at

$$
\left|T_{11}^{-}\right|_{\min }=92.4996(\mathrm{KPa}) .
$$

There is only a group of solutions in which the solution like $\left[N_{1}>0, N_{2}>0, f_{1}<0, f_{2}>0\right]^{\mathrm{T}}$ takes the following values

$$
\left\{\begin{array}{l}
N_{1} \\
N_{2} \\
f_{1} \\
f_{2}
\end{array}\right\}=\left\{\begin{array}{c}
5.74821628506585 \times 10^{-1} \\
8.18278739428709 \times 10^{-1} \\
-5.74611511535824 \times 10^{-2} \\
7.44451697097634 \times 10^{-2}
\end{array}\right\} .
$$

The predicted $\left|T_{11}^{-}\right|_{\min }$ value is $92.4996 \mathrm{KPa}$. Then, we can obtain stresses as follow, which are stresses inside the shear band

$$
\left(\pi^{+}\right)^{\mathrm{T}}=\left[\begin{array}{ccc}
-94.2503 & -2.46965 & 0 \\
2.16782 & -13.3125 & 0 \\
0 & 0 & -45.5710
\end{array}\right](\mathrm{KPa}),
$$

and stresses outside the band 


$$
\left(\pi^{-}\right)^{\mathrm{T}}=\left[\begin{array}{ccc}
-91.1643 & 0 & 0 \\
0 & -15.0474 & 0 \\
0 & 0 & -29.0248
\end{array}\right](\mathrm{KPa}) .
$$

And we obtain strains as follow, which are strains inside the shear band

$$
\mathbf{E}^{+}=\left[\begin{array}{ccc}
-0.0454232 & 0.000373662 & 0 \\
0.000373662 & 0.0672368 & 0 \\
0 & 0 & 0
\end{array}\right],
$$

and strains outside the band

$$
\mathbf{E}^{-}=\left[\begin{array}{ccc}
-0.0143311 & 0 & 0 \\
0 & 0.00316651 & 0 \\
0 & 0 & 0
\end{array}\right] .
$$

And we obtain deformation gradients inside the shear band

$$
\boldsymbol{F}^{+}=\left[\begin{array}{ccc}
0.952534 & -0.0470192 & 0 \\
0.0427926 & 1.06407 & 0 \\
0 & 0 & 1
\end{array}\right],
$$

and deformation gradients outside the band

$$
\boldsymbol{F}^{-}=\left[\begin{array}{ccc}
0.985565 & 0 & 0 \\
0 & 1.00316 & 0 \\
0 & 0 & 1
\end{array}\right] .
$$

By Eq. (1) we have the inclination angle

$$
\theta=54.9128^{\circ},
$$

which is agreed well with value of $57^{\circ}$ measured in [10] with error of $3.7 \%$.

\section{Conclusions}

Strain localization is the event of two-phase coexistence that can be observed in sand with strain softening. The theory on multi-phase equilibrium is suitable for the analysis of its formation. The analysis in the present paper shows that locally-deformed bands can certainly occur in sand, both left-upward shear bands and right-upward shear bands have been predicted as had observed in experiments, the predicted inclination angle of the band and the stress for two-phase coexistence are in good agreement with the experimentally measured values.

\section{Acknowledgements}

This study was supported by the Natural Science Foundation of Hebei Province, China (Grant No. A2015202242 and No. F2015202214) and Natural Science Foundation of Tianjin, China (Grant No. 16JCQNJC00400).

\section{References}

1. K.H. Roscoe, Geotechnique 20, 129-170 (1970)

2. I. Vardoulakis, Mech. Res. Commun. 3, 209-214 (1976)

3. J.R.F. Arthur, T. Dunstan, Q.A.J.L. Al-Ani, A. Assadi, Geotechnique 27, 53-74 (1977)

4. I. Vardoulakis, B. Graf, Geotechnique 35, 299-317 (1985) 
5. M. Mokni, J. Desrues, Mech. Cohes-Frict. Mat. 4, 419-441 (1999)

6. A. Drescher, I. Vardoulakis, C. Han, Geotech. Test. J. 13, 226-234 (1990)

7. C. Han, I. Vardoulakis, Geotechnique 41, 49-78 (1991)

8. W.W. Harris, G. Viggiani, M.A. Mooney, R.J. Finno, Geotech. Test. J. 18, 405-420 (1995)

9. R.J. Finno, W.W. Harris, M.A. Mooney, G. Viggiani, J. Geotech. Eng. 122, 462-473 (1996)

10. K.A. Alshibli, S. Sture, J. Geotech. Geoenviron. 126, 495-503 (2000)

11. R.C.K. Wong, Int. J. Numer. Anal. Meth. Geomech. 25, 131-153 (2001)

12. A.L. Rechenmacher, J. Mech. Phys. Solids. 54, 22-45 (2006)

13. J. Desrues, G. Viggiani, Int. J. Numer. Anal. Meth. Geomech. 28, 279-321 (2004)

14. D. Qi, Y. Zhang, J. Ning, T. Huang, Acta. Mech. Solida Sin. 24, 166-175 (2011)

15. Y. Zhang, S. Ren, T. Ao, Mat. Sci. Eng. A-Struct. 447, 332-340 (2007)

16. Y.B. Fu, A.B. Freidin, Proc. R. Soc. Lond. A 460, 3065-3094 (2004)

17. D.C. Drucker, W. Prager, Quart. Appl. Math. 10, 157-165 (1952) 